\title{
Performance of OFDM System under Different Fading Channels and Coding
}

\author{
Pratima Manhasa*1, M. K. Sonib ${ }^{2}$ \\ ${ }^{1}$ FET, ECE Dept, Manav Rachna International University, Faridabad, India \\ ${ }^{2}$ ED\& Dean, FET, Manav Rachna International University, Faridabad, India \\ ${ }^{\star}$ Corresponding author, email: pratimamehak@gmail.com* , ed.fet@mriu.edu.in ${ }^{2}$
}

\begin{abstract}
Orthogonal frequency division multiplexing (OFDM) is a type of multicarrier modulation (MCM) technique in which larger bandwidth is divided into parallel narrow bands each of which is modulated by different subcarriers. All the subcarriers are orthogonal to each other and hence it reduces the interference among various subcarriers. OFDM technique is an efficient modulation technique used in certain wired and wireless application.In a wireless communication channel, the transmitted signal can travel from transmitter to receiver over multiple reflective paths. This results to multipath fading which causes fluctuations in amplitude, phase and angle of arrival of the received signal. For example, the signal which is transmitted from BTS (base transceiver station) may suffer multiple reflections from the buildings nearby, before reaching the mobile station. Such multipath fading channels are classified into slow fading/fast fading and frequency-selective/flat fading channels. This paper discusses the performance of OFDM system using various fading channels and channel coding. The parameter which is known as Bit error rate (BER) is calculated under different fading channels (AWGN, Rayleigh and Rician) for different digital modulation (BPSK, QPSK and QAM) and Channel coding (linear/Cyclic coding). Matlab Simulink tool is used to calculate the BER parameter.
\end{abstract}

Keywords: Fading, Rayleigh, Rician, Linear, Cyclic, Bit error rate, Simulink

\section{Introduction}

OFDM is an efficient digital modulation technique which is widely used in both wired and wireless communication system. For wired application it is used in DSL, ADSL and for wireless application it is used in wireless LAN. It is a bandwidth efficient technique in which larger bandwidth is divided into parallel narrow bands each of which is modulated by different subcarriers. OFDM has high peak to average power ratio and synchronization errors. The performance of BER is calculated by using different fading channel and channel coding [1].

\subsection{Fading Channel}

Fading generally is a signal loss either in amplitude or phase due to sudden changes in Channel response. The fading is considered to be a random process which may vary with time, geographical position or radio frequency. In wireless systems, fading may either be due to multipath propagation, referred to as multipath induced fading, or due to shadowing from obstacles affecting the wave propagation, sometimes referred to as shadow fading.Large scale fading (Shadowing) concerns about large distances effect so, its affect appears clearly incase of the displacement of either the Transmitter or the Receiver. Small scale fading is concerned about very small changes in the position of Transmitter or receiver in order of the wavelength [2]. A model is required to predict the effects of this fading accurately in order to mitigate it effects. Some of the channels used to model multipath fading are:

\subsubsection{AWGN Channel}

An AWGN channel results in addition of white Gaussian noise to the signal that passes through it. The AWGN Channel block adds white Gaussian noise to a real or complex input signal. When the input signal is real, the AWGN channel block adds real Gaussian noise and produces a real output signal. When the input signal is complex, this AWGN channel block adds complex Gaussian noise and produces a complex output signal. 


\subsubsection{Rayleigh Channel}

Rayleigh fading is a model that can be used to describe the form of fading that occurs when multipath propagation exists. In any terrestrial environment a radio signal will travel through a number of different paths from the transmitter to the receiver. The clearest path is the direct, or line of sight path [3]. The Rayleigh fading model can be used to analyze radio signal propagation on a statistical basis. It operates best under conditions when there is no dominant signal (e.g. direct line of sight signal) and in many instances cellular telephones being used in a dense urban environment fall into this category. Other examples where no dominant path generally exists are for ionospheric propagation where the signal reaches the receiver through a huge number of individual paths. Propagation using tropospheric ducting also exhibits the same patterns.

\subsubsection{Rician Channel}

Fading causes the signal to spread and become diffuse. The Rician Fading Channel block implements a baseband simulation of a Rician fading propagation channel.This block is useful for modeling mobile wireless communication systems when the transmitted signal can travel to the receiver along a dominant line-of-sight or direct path.The K-factor parameter is used for Rician distribution which represents the ratio between direct-path (unspread) power and diffuse power. The ratio is expressed linearly, not in decibels. While the Gain parameter controls the overall gain through the channel, the K-factor parameter controls the gain's partition into direct and diffuse components.

\subsection{Channel Coding}

Source encoding is used to convert the message in the coded form to be transmitted through the channel and channel encoding deals with source encoded message by introducing some extra bits that will be used in the detecting \& correcting error [5].

The channel encoding methods can be classified as:

a. Linear Block codes

Block code is a type of error correcting code in which "k" bits input data is used to produce" n" bits of output data. It is notated as (n, k). Linear block codes are so named because each code word in the set is a linear combination of a set of generator code words [6]. Any message of $k$ bits can be generated by multiplication of message vector by the generator matrix to produce a code word vector that is $n$ bits long shown in equation (1). The product of $m$ and $G$ gives the code word generation. Code word generation $(C)$ is given as

$$
C=m^{*} G
$$

Where $m$ is message and $G$ is generator It is shown in Figure 1

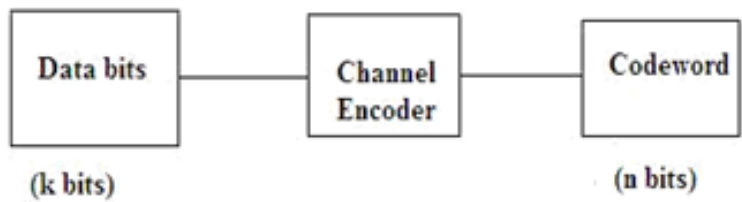

Figure 1. Representation of linear block code

In linear block code, the message is divided into blocks of $\mathrm{k}$ bits. Each block is encoded to a larger block of $n$ bits. The $n$ bit codeword includes message and parity bits. After this the coded bits are modulated and sent over channel. The reverse procedure is done at the receiver. The block diagram (Figure 2) represents the data bits ( $k$ bits) and after passing through the channel encoder is converted into $\mathrm{n}$ bit codeword[7]. 


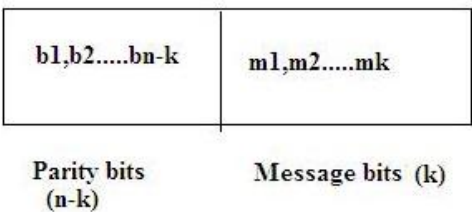

Figure 2. Block diagram of linear block code

b. Cyclic block codes

They can be easily implemented in hardware. They can be determined algebraically and hence can be decoded in constant time. They are very useful in situations where the BER of the channel is relatively low, bandwidth availability is limited in the transmission, and it is easy to retransmit data [14].

\section{Proposed Simulink Model}

The simulink model for OFDM system using various fading channels (AWGN, Rayleigh and Rician), channel coding (Linear/Cyclic) and various digital modulations (BPSK, QPSK and QAM) has the following steps and it is shown in Figure 3.

1) Bernoulli binary generator is used to generate the input signal. After getting the input signal various channel coding is applied. Then the channel coded input signal is mapped by using various modulations (BPSK/QPSK and QAM).

2) The mapped output is modeled using Inverse Fast Fourier transform (IFFT).

3) The output of Inverse Fast Fourier transform is passed through the AWGN, Rayleigh and Rician channel and then demodulation is done to calculate the BER parameter using Error rate calculation [7].

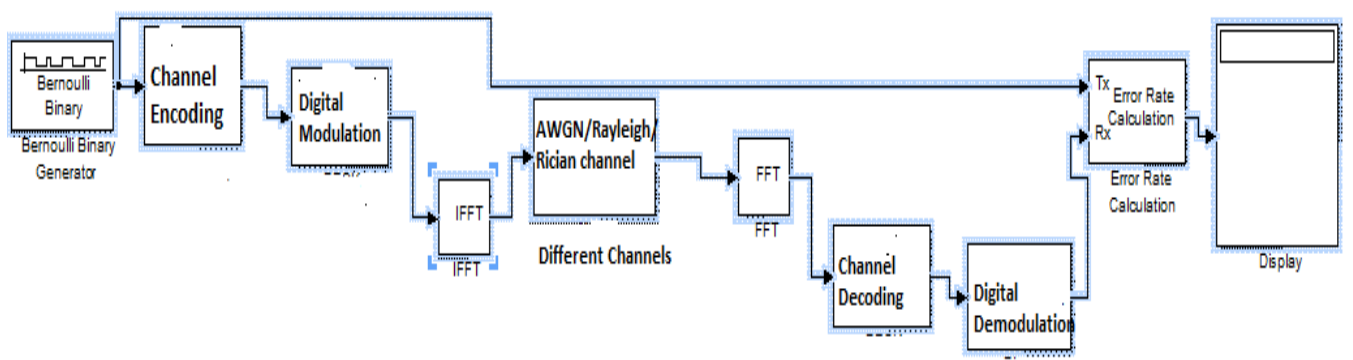

Figure 3. Proposed simulink model using different coding and channel

\subsection{Proposed OFDM model using AWGN Channel with Linear/Cyclic coding}

The proposed simulink model using AWGN channel including different channel coding is shown in Figure 4. In the given model Bernoulli binary generator is used to generate the input signal and then different coding is applied (linear/cyclic). The coded output is modulated by using different digital modulation. Inverse fast Fourier transform is applied on the modulated output and then the signal is passed through AWGN channel and reverse operation is performed at the receiver side. 


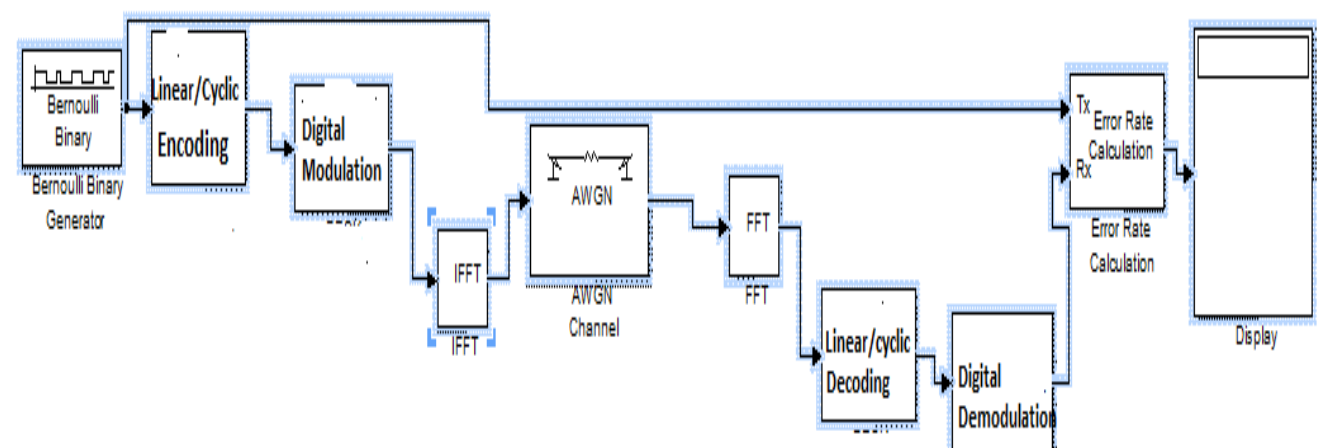

Figure 4. Proposed simulink model using different coding and AWGN channel

\subsection{Proposed OFDM Model Using Rayleigh Channel with Linear/Cyclic Coding}

The Proposed simulink model using Rayleigh fading channel using Linear/Cyclic coding is shown in Figure 5. In the given model Bernoulli binary generator is used to generate the input signal and then different coding is applied (linear/cyclic). The coded output is modulated by using different digital modulation. Inverse fast Fourier transform is applied on the modulated output and then the signal is passed through Rayleigh fading channel and reverse operation is performed at the receiver side.

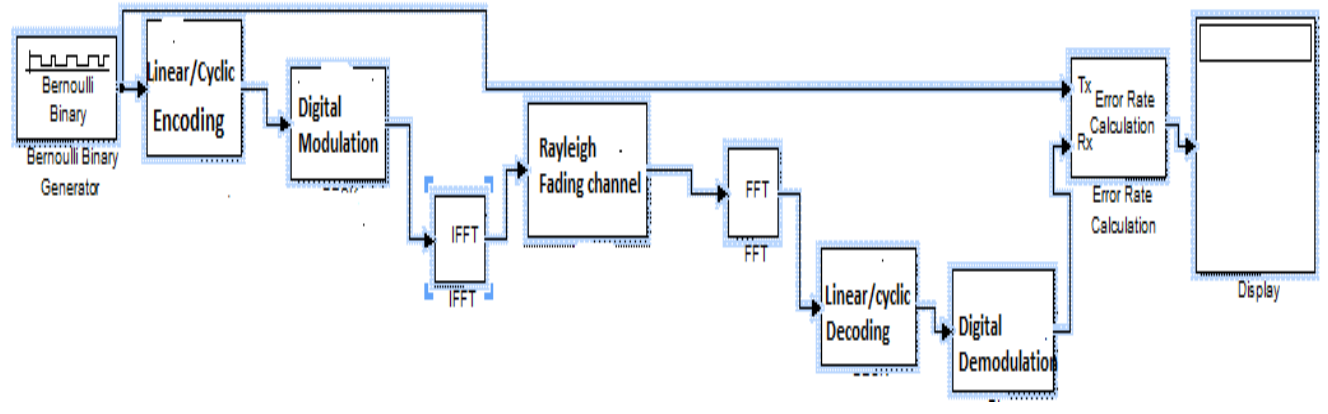

Figure 5. Proposed simulink model using different coding and Rayleigh channel

\subsection{Proposed OFDM Model Using Rician Channel with Linear/Cyclic coding}

The Proposed model using the Rician fading channel using Linear/Cyclic coding is shown in Figure 6. In the given model Bernoulli binary generator is used to generate the input signal and then different coding is applied (linear/cyclic). The coded output is modulated by using different digital modulation. Inverse fast Fourier transform is applied on the modulated output and then the signal is passed through Rician fading channel and reverse operation is performed at the receiver side. 


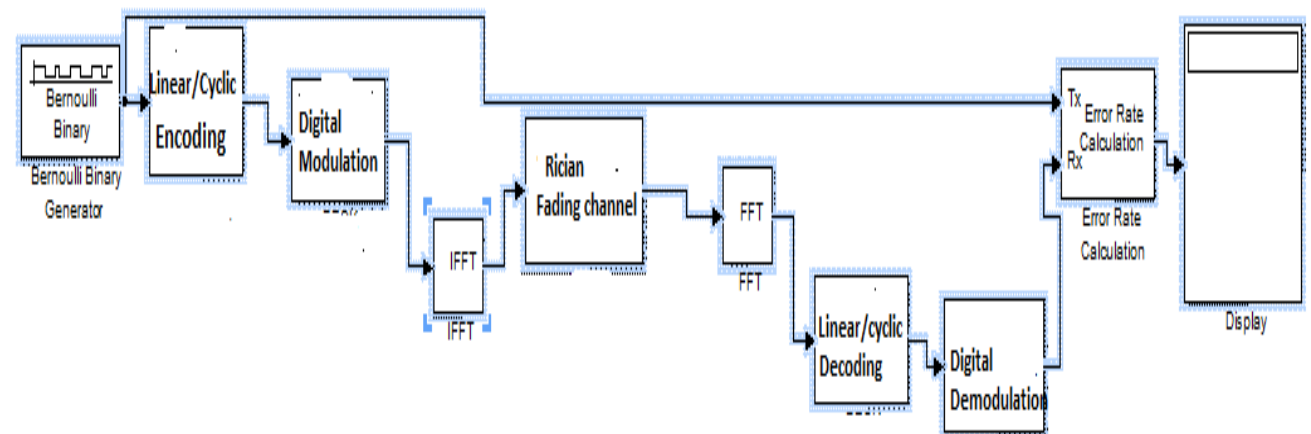

Figure 6. Proposed simulink model using different coding and Rician channel

\section{Simulation Results}

The simulation parameters which are used for the proposed work are given below:

Digital ModulationBPSK, QPSK and QAM

Fading ChannelAWGN, Rayleigh and Rician

Channel codingLinear, cyclic

The BER results of OFDM system using various fading channel and channel coding are shown in Figures7-9. In Figure 7, the BER result using BPSK based system results in minimum value of BER as compared to QPSK and QAM based system.

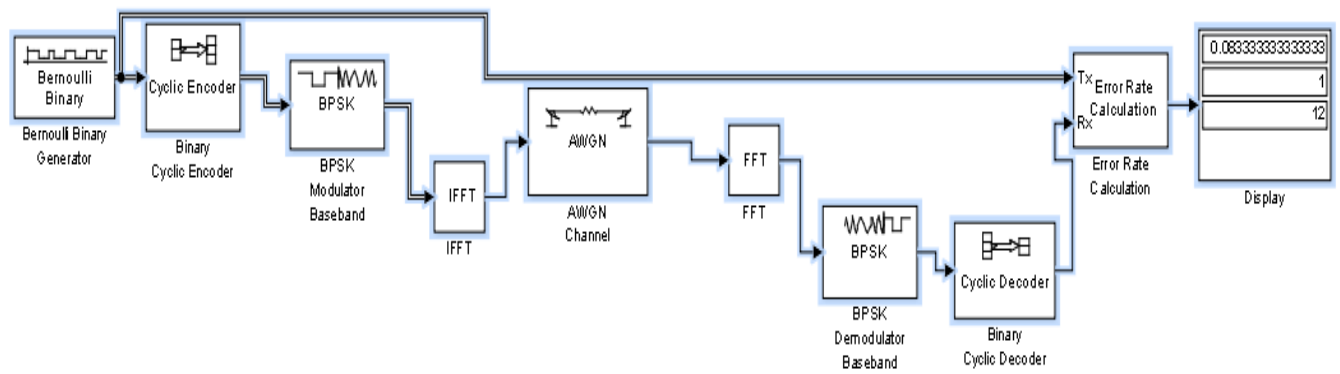

Figure 7. BER results of OFDM system using different coding and AWGN channel

The BER results using BPSK modulation for Rayleigh channel coding is shown in Figure 8. In case of Rayleigh fading channel, BPSK based OFDM system results in minimum value of BER as compared with QPSK and QAM based system.

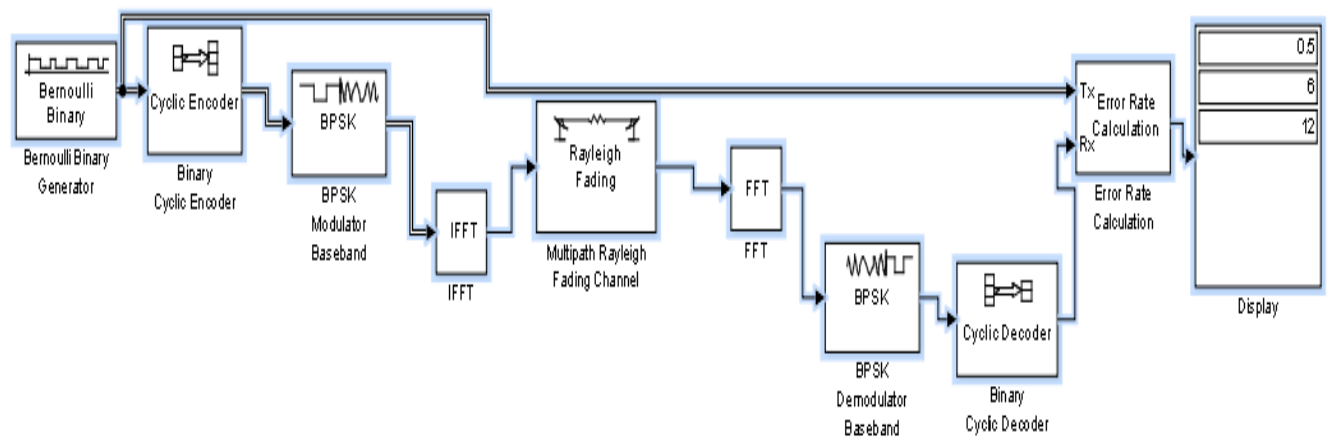

Figure 8. BER results of OFDM system using different coding and Rayleigh channel 
The BER results using BPSK modulation for Rician channel coding is shown below in Figure 9. In case of Rician fading channel, BPSK based OFDM system results in minimum value of BER as compared with QPSK and QAM based system.

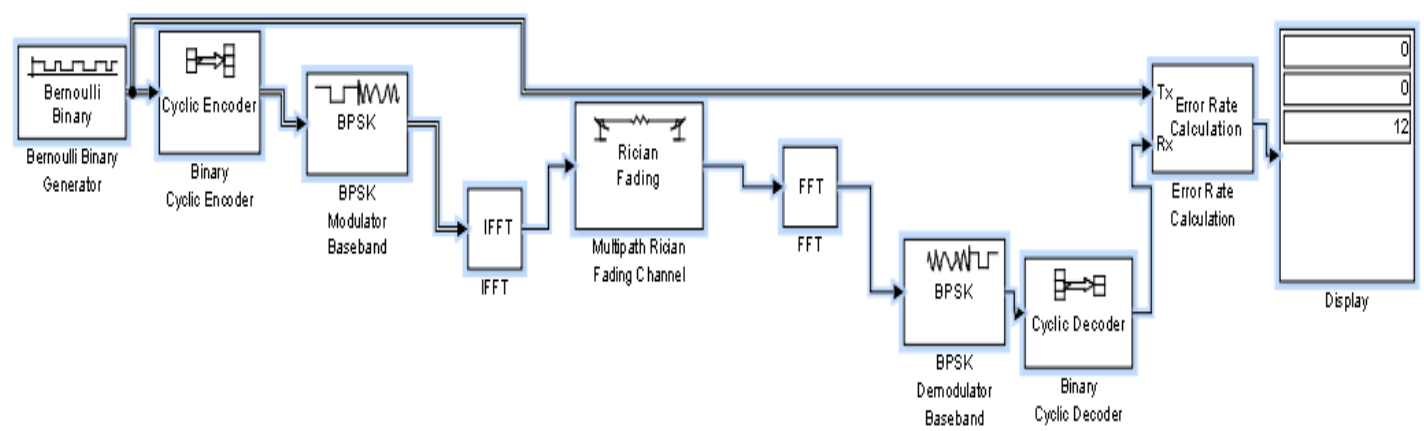

Figure 9. BER results of OFDM system using different coding and Rician channel

The BER calculation of FFT based OFDM system for different channel coding and fading channel are shown in below in Table 1, 2 and 3 and bar chart is represented in Figures 10,11 , and 12.

Table 1. BER results of FFT Based OFDM System with Different Fading Channels and without Channel Coding

\begin{tabular}{cccc}
\hline $\begin{array}{c}\text { OFDM system with } \\
\text { different modulation }\end{array}$ & $\begin{array}{c}\text { AWGN } \\
\text { channel }\end{array}$ & $\begin{array}{c}\text { Rayleigh } \\
\text { Channel }\end{array}$ & $\begin{array}{c}\text { Rician } \\
\text { Channel }\end{array}$ \\
\hline BPSK & 0.272 & 0.454 & 0 \\
QPSK & 0.3636 & 0.545 & 0.545 \\
QAM & 0.636 & 0.6363 & 0.909 \\
\hline
\end{tabular}

The Table 1 compares the BER results of FFT based OFDM system without channel coding for different fading channel. From Table 1, it is clear that using AWGN channel, the BER results are minimum and BPSK modulation also results in minimum value of BER. So, the BPSK modulation with AWGN channel results in reducing the value of BER. The bar chart for Table 1 is shown in Figure 10.

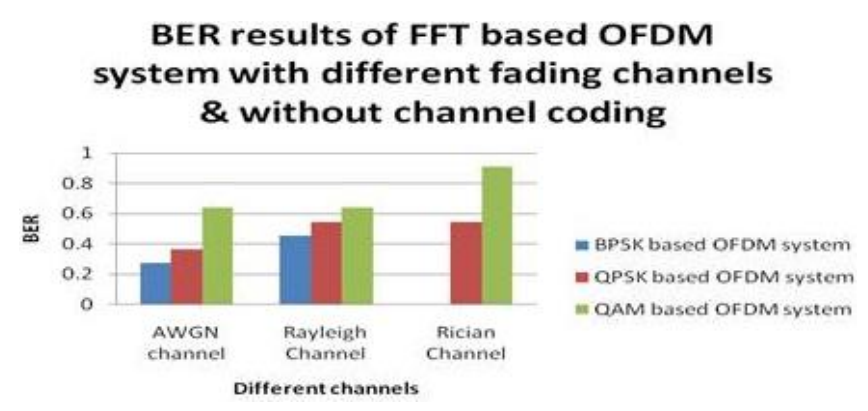

Figure 10. BER graph for various fading channel and without channel coding

Table 2, represents the BER results for FFT based OFDM system using AWGN, Rayleigh and Rician channel for linear coding. From the given Table 2, the results of BER for AWGN channel for BPSK modulation are better as compared with Rayleigh and Rician. After AWGN channel, the results of Rician channel is better than Rayleigh channel. 
Table 2. BER Results of FFT based OFDM System with Different Fading Channels and Linear Coding

\begin{tabular}{cccc}
\hline $\begin{array}{c}\text { OFDM system with } \\
\text { different modulation }\end{array}$ & $\begin{array}{c}\text { AWGN } \\
\text { channel }\end{array}$ & $\begin{array}{c}\text { Rayleigh } \\
\text { Channel }\end{array}$ & $\begin{array}{c}\text { Rician } \\
\text { Channel }\end{array}$ \\
\hline BPSK & 0 & 0.5 & 0 \\
QPSK & 0.0833 & 0.33 & 0 \\
QAM & 0.5833 & 0.916 & 0.916 \\
\hline
\end{tabular}

The bar chart for above Table 2 is shown in Figure 11. The bar chart represents the $\mathrm{BER}$ results for different fading channel with linear coding.

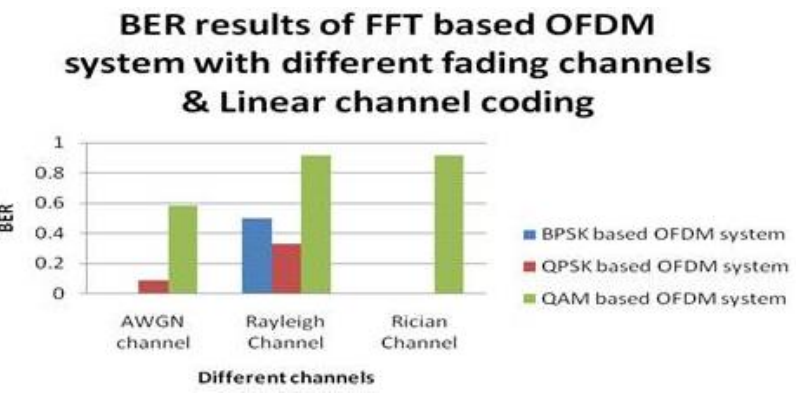

Figure 11. BER graph for various fading channel and linear channel coding

Table 3, represents the BER results for FFT based OFDM system using AWGN, Rayleigh and Rician channel for cyclic coding. From the given Table 3, the results of BER for AWGN channel for BPSK modulation are better as compared with Rayleigh and Rician. After AWGN channel, the results of Rician channel is better than Rayleigh channel.

Table 3. BER Results of FFT Based OFDM System with Different Fading Channels and

\begin{tabular}{cccc}
\multicolumn{4}{c}{ cyclic Coding } \\
\hline $\begin{array}{c}\text { OFDM system with } \\
\text { different modulation }\end{array}$ & $\begin{array}{c}\text { AWGN } \\
\text { channel }\end{array}$ & $\begin{array}{c}\text { Rayleigh } \\
\text { Channel }\end{array}$ & $\begin{array}{c}\text { Rician } \\
\text { Channel }\end{array}$ \\
\hline BPSK & 0.083 & 0.5 & 0 \\
QPSK & 0.083 & 0.166 & 0.083 \\
QAM & 0.416 & 0.916 & 0.916 \\
\hline
\end{tabular}

The bar chart for above Table 3 is shown in Figure 12. The bar chart represents the BER results for different fading channel with cyclic coding.

\section{BER results of FFT based OFDM system with different fading channels \& Cyclic channel coding}

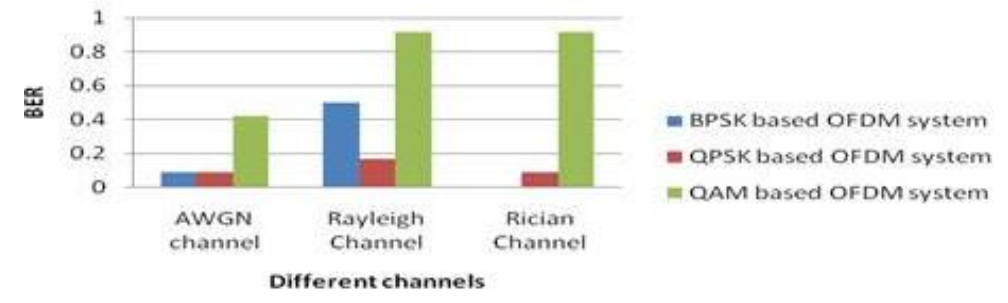

Figure 12. BER graph for various fading channel and cyclic channel coding 
Simulation results show that cyclic coding FFT based OFDM system results in minimum value of BER as compared to Linear coding. For different fading channels, AWGN channel results in minimum BER as compared to Rayleigh and Rician channel. The BER value using Rayleigh channel is more than Rician and AWGN. The OFDM system using BPSK modulation with cyclic coding and AWGN channel results in minimum BER.QPSK based OFDM system using cyclic coding for AWGN and Rician fading channel results in the same value of BER but has higher value of BER for Rayleigh channel. So in order to model FFT based OFDM system, cyclic coding and AWGN channel results in minimum value of BER.

\section{Conclusion}

The performance of FFT based OFDM system using various channels and channel coding is simulated using simulink model. The results show that the BER is better in AWGN channel and worst in Rayleigh channel. Rician fading channel has better BER value than Rayleigh but less than AWGN channel. The cyclic coding based OFDM system with AWGN channel helps in reducing the BER value.

\section{References}

[1] Heiskala J, Terry Ph D, J. OFDM wireless LANs: A theoretical and practical guide. Sams. 2001.

[2] Nee RV, Prasad R. OFDM for wireless multimedia communications. Artech House, Inc. 2000.

[3] Jiang T, Wu Y. An overview: peak-to-average power ratio reduction techniques for OFDM signals. IEEE transactions on Broadcasting. 2008; 54(2): 257.

[4] Kaiser S. OFDM code-division multiplexing in fading channels. Communications, IEEE Transactions. 2002; 50(8): 1266-1273.

[5] Sanjiv Kumar, PK Gupta Singh, Chauhan DS. Performance analysis of Rayleigh and Rician fading channel models using Matlab simulation. I.J intelligent systems and applications. 2013; 9: 94-102.

[6] Fazel K. Performance of CDMA/OFDM for mobile communication system. In Universal Personal Communications, 1993. Personal Communications: Gateway to the 21st Century. IEEE, Conference Record, 2nd International Conference on October 1993; 2: 975-979.

[7] Manhas P, Soni MK. Comparison of OFDM System in terms of BER using Different Transform and Channel Coding. I.J engineering and manufacturing. 2016; 1: 28-34.

[8] Burr A, Kandus G. 2.3. Modulation and Coding. Wireless Flexible Personalised Communications. 2001: 51.

[9] Ergen M. Principles of OFDM. In Mobile Broadband. Springer US. 2009: 109-175.

[10] Agarwal A, Patra SK. Performance prediction of OFDM based DAB system using block coding techniques. In Emerging Trends in Electrical and Computer Technology (ICETECT). IEEE, International Conference. March 2011: 792-796.

[11] Agarwal A. Digital Audio Broadcast: Modulation, Transmission \& Performance Analysis (Doctoral dissertation, National Institute of Technology Rourkela) 2010.

[12] Malhotra J. Investigation of Channel Coding Techniques for High Data Rate Mobile Wireless Systems. International Journal of Computer Applications. 2015: 115(3).

[13] Rugini L, Banelli P. BER of OFDM systems impaired by carrier frequency offset in multipath fading channels. Wireless Communications, IEEE Transactions. 2005; 4(5): 2279-2288.

[14] Manhas P, Soni MK. 2015. OFDM PAPR Reduction Using Recurring SLM with Cyclic and Linear Block Codes Schemes. TELKOMNIKA Indonesian Journal of Electrical Engineering. 2015; 16(1): 1-6. 